\title{
Clinicopathological Correlation in Patients of Lymphadenopathy in Tertiary Care Hospital
}

\author{
Dr. Amol R. Rajhans ${ }^{* 1}$, MD, Dr. Deepak S. Howale ${ }^{2}$ \\ ${ }^{1}$ Pathology, DCP Consultant Pathologist Shashwat Hospitals, Pune \\ ${ }^{2}$ Dean Govt. Medical College, Silvassa
}

\begin{abstract}
Lymph nodes are parts of the lymphatic system. Lymph node helps to filter out viruses, bacteria, cancer cells and other unwanted substances safely removed from the body. Lymphadenopathy is common clinical problem frequently give dilemma in diagnostic. Enlargement of lymph node is a common problem which evaluate as a Lymphadenopathy. An abnormality in size and character of lymph node in known as Lymphadenopathy which also consider as enlarged abnormally that measures more than $10 \mathrm{~mm}$ in its diameter than normal. Lymphadenopathy is common clinical problem frequently give dilemma in diagnostic. Fine needle aspiration cytology (FNAC) has become an important tool for initial diagnosis and management for patients suffering from Lymphadenopathy because of early result, simple for test and minimal trauma to patient. The main aim of this study is to Correlation of Clinicopathological condition presenting with Lymphadenopathy. Role of FNAC in diagnosis will also evaluate with node biopsy and open lymph. Material and methods: Total 100 patients were included in this study. For all 100 patients fine needle aspiration cytology (FNAC) was done. In 75 cases excision biopsy was done. Pathological diagnosis was obtained in all cases. Fine needle aspiration cytology, excision biopsy, throat, ear and nose examination were carried out as a required base line investigations. Result:Total 80 patients were studies in this study. Out of this 80 patients 30 cases were IPD and remaining were OPD.FNAC as well as biopsy were carried out from total patients in this study. In this study Tubercular Lymphadenitis was observer maximum. Out of 80 patients 47(58.75\%) were male and 33(41.25\%) were female patients. Age group 20-30 years (35\%) were maximum number of patients which was followed by $30-40$ years $(23.75 \%)$ and $10-20$ years $(21.25 \%)$ respectively was observed. Out of 80 patients $50(62.5 \%)$ were diagnosis as Tubercular Lymphadenitis and Chronic Non-specific Lymphadenitis was diagnosis in 10 (12.5\%). Reactive lymphadenitis was diagnosed in $8(10 \%)$ patients whereas Malignant secondary's were diagnosed in $7(8.75 \%)$ patients followed by $3(3.75 \%)$ patients suffer from lymphomas and 2(2.5\%) were Non- Hodgkin's Lymphoma. Conclusion: Lymphadenopathy is a clinical evaluation followed by FNAC which is reliable diagnostic tool that is easy to perform; cost effective, speedy results accurate can be obtained. Specialist input is the main requirement for this. Biopsy is also useful in cases of lymphomas that act as diagnostic tool. When FNAC report is inconclusive, in nonspecific lymphadenitis than Biopsy is much helpful for accurate diagnosis and management. Hence, in cases of Lymphadenopathy FNAC was found to be reliable, simple and cost effective method for diagnosis.
\end{abstract}

Keywords: Lymphadenopathy, FNAC, Biopsy, Tubercular Lymphadenitis

\section{Introduction}

Lymphadenopathy is common clinical problem frequently give dilemma in diagnostic. Lymph nodes are parts of the lymphatic system. Lymph node helps to filter out viruses, bacteria, cancer cells and other unwanted substances and safely removed from the body.This may be primary or secondary characteristics of numerous disease. ${ }^{[1]}$ Enlargement of lymph node is a common problem which evaluate as a Lymphadenopathy. An abnormality in size and character of lymph node in known as Lymphadenopathy which also consider as enlarged abnormally that measures more than $10 \mathrm{~mm}$ in its diameter than normal. Palpable supraclavicular nodes are known as abnormal and consider as Lymphadenopathy. These are because of being self-limiting disease in which self limited bacterial and viral infections are common causes. ${ }^{[2]}$ Lymphadenopathy may affect in any age group, but it is common in children and young adults whereas in older age group it may be due to secondary metastasis.Malignancies like leukemia and lymphoma there is enlargement of cervical nodes as an initial presentation. There are various differential diagnoses of lymphadenothathy and there evaluation and management.
Fine needle aspiration cytology (FNAC) has become an important tool for initial diagnosis and management for patients suffering from Lymphadenopathy because of early result, simple for test and minimal trauma to patient.This test is quick, reliable cost effective and safe test for routine diagnosis. The diagnosis of metastatic tumour for lymph node would be single criteria for determining primary tumour especially in occult primary cases. ${ }^{[3]}$

Analysis of cervical Lymphadenopathy is never straightforward and difficult in differential diagnoses that resemble with several diseases which include neoplastic disorders and inflammatory. ${ }^{[4,5,6,7]}$ Aspiration cytology, open biopsy and clinical evaluation are some methods for diagnosis of Lymphadenopathy. Every method has its own advantages and disadvantages. Currently histopathology examination is gold standard for diagnosis of Lymphadenopathy. ${ }^{[8,9]}$

The main aim of this study is to Correlation of Clinicopathological condition presenting with Lymphadenopathy. Role of FNAC in diagnosis will also evaluate with node biopsy and open lymph. 
International Journal of Innovative Research in Medical Science (IJIRMS)

Volume 03 Issue 12 Dec 2018, ISSN: 2455-8737, Imp. Factor - 4.102

Available online at - $\underline{w w w . j j i r m s . i n}$

\section{Material and methods}

The study was conducted in Department of pathology at Shashwat Hospital Pune. Patients with significant Lymphadenopathy attending OPD and IPD patients were included in this study.Total 100 patients were included in this study. For all 100 patients fine needle aspiration cytology (FNAC) was done. In 75 cases excision biopsy was done. Pathological diagnosis was obtained in all cases.

Fine needle aspiration cytology, excision biopsy, throat, ear and nose examination were carried out as a required base line investigations.

\section{Result}

Total 80 patients were studies in this study. Out of this 80 patients 30 cases were IPD and remaining were OPD.FNAC as well as biopsy were carried out from total patients in this study. In this study Tubercular Lymphadenitis was observer maximum. Out of 80 patients $47(58.75 \%)$ were male and $33(41.25 \%)$ were female patients as shown in table 1 .

Table No 1: Sex distribution of Patients

\begin{tabular}{|l|c|c|}
\hline Sex & No & Percentage \\
\hline Male & 47 & 58.75 \\
\hline Female & 33 & 41.25 \\
\hline Total & 80 & 100 \\
\hline
\end{tabular}

In this study it was observed that patients with age group 20-30 years $(35 \%)$ were maximum number of patients which was followed by $30-40$ years $(23.75 \%)$ and $10-20$ years $(21.25 \%)$ respectively as shown in table no 2 . In all age groups tuberculosis was diagnosed with more number of cases and in the age groups of 20 to 30 years and 30 to 40 years. Malignant secondary's were clustered in patients above the age of 50 years.

Table no.2: Age and sex distribution.

\begin{tabular}{|l|c|c|}
\hline Age group & No & Percentage \\
\hline $\mathbf{1 0 - 2 0}$ & 17 & 21.25 \\
\hline $\mathbf{2 0 - 3 0}$ & 28 & 35 \\
\hline $\mathbf{3 0 - 4 0}$ & 19 & 23.75 \\
\hline $\mathbf{4 0 - 5 0}$ & 9 & 11.25 \\
\hline $\mathbf{5 0 - 6 0}$ & 7 & 8.75 \\
\hline Total & 80 & 100 \\
\hline
\end{tabular}

In this study tubercular Lymphadenitis is more common. Out of 80 patients 50(62.5\%) were diagnosis as Tubercular Lymphadenitis. Chronic Non-specific Lymphadenitis was diagnosis in 10 (12.5\%) patients. Reactive lymphadenitis was diagnosed in $8(10 \%)$ patients.Malignant secondary's were diagnosed in $7(8.75 \%)$ patients whereas $3(3.75 \%)$ patients suffer from lymphomas and 2(2.5\%) were Non- Hodgkin's Lymphoma. Therefore among the various causes for Lymphadenopathy, tuberculosis was the most common cause as shown in table no. 3

Table no. 3: Histopathological diagnosis

\begin{tabular}{|l|c|c|}
\hline Cytology & No & Percentage \\
\hline Tubercular Lymphadentitis & 50 & 62.5 \\
\hline Reactive lymphadentitis & 8 & 10 \\
\hline Secondaris & 7 & 8.75 \\
\hline
\end{tabular}

\begin{tabular}{|l|c|c|}
\hline Chronic Non-specific Lymphadentitis & 10 & 12.5 \\
\hline Hodgkin's Lymphoma & 3 & 3.75 \\
\hline Non- Hodgkin's Lymphoma & 2 & 2.5 \\
\hline Total & 80 & 100 \\
\hline
\end{tabular}

In this study, as shown in table no 4 incidence of presenting symptoms neck swelling was most common presenting complaint. Out of $80,38(47.5 \%)$ cases were shows the presentation of neck swelling, followed by fever 15(18.75\%), loss of weight $6(7.5 \%)$ and both malaise and loss of appetiteis $4(5 \%)$ respectively.

Table no.4: Incidence of presenting symptoms

\begin{tabular}{|l|c|c|}
\hline Presenting symptoms & No & Percentage \\
\hline Neck swelling & 38 & 47.5 \\
\hline Fever & 15 & 18.75 \\
\hline Cough & 12 & 1.25 \\
\hline Change of voice & 1 & 15 \\
\hline Malaise & 4 & 5 \\
\hline Loss of appetite & 4 & 5 \\
\hline Loss of weight & 6 & 7.5 \\
\hline Total & 80 & 100 \\
\hline
\end{tabular}

\section{Discussion}

In this study, data has been analyzed with age, sex, clinical findings, symptoms and presenting complaints and result of FNAC and biopsy.In this study, 50(62.5\%) have tuberculosis lymphadenitis, where as Chronic Non-specific Lymphadenitis was diagnosis in $10(12.5 \%)$ patients. Reactive lymphadenitis was diagnosed in $8(10 \%)$ patients.Malignant secondary's were diagnosed in $7(8.75 \%)$ patients whereas $3(3.75 \%)$ patients suffer from lymphomas and 2(2.5\%) were Non- Hodgkin's Lymphoma which are almost similar to the other studies as Dandapat et.al., ${ }^{[10]}$ Das gupta et.al., ${ }^{[11]}$ mondal A et.al., ${ }^{[12]}$ and Prasad RR et.al., ${ }^{[13]}$ The cervical lymphadenitis is most common presentation of swelling in the neck. $74 \%$ patients were diagnosed as tuberculous etiology as in study of Gupta et.al., ${ }^{[14]}$

In the present study, out of the 80 patients, 50 patients were diagnosed to have tuberculosis and $35 \%$ i.e., 28 cases were of the age group of 20-30 years which was similar to study of Panditetal., ${ }^{[15]}$ of which the mean age of presentation was 20-30 years age group. In the study by Rakshanetal., ${ }^{[16]}$ the mean age was 26 years, where as in the study of Narang et.al. ${ }^{[17]}$ the mean age of presentation was 25 years. The findings of our study compare well with these studies.

In present study, incidence of presenting symptoms neck swelling was most common presenting complaint were observed.47.5\% cases were observed with symptom of neck swelling, followed by fever $18.75 \%$, loss of weight $7.5 \%$ and both malaise and loss of appetite is $5 \%$ respectively which was almost equivalent to other studies Khan et al., ${ }^{[18]}$ Desai et.al., ${ }^{[19]}$

According to study of Ahmed et.al., ${ }^{[20]}$ and Hirachand et.al., ${ }^{[21]}$ observed an incidence of $12 \%$ for secondary's and incidence of $12 \%$. The clinical diagnosis was based on lymph node, their consistency, immobile and fixed. Therefore, FNAC diagnosis turned about $90 \%$ of clinical diagnosis which is 


\section{International Journal of Innovative Research in Medical Science (IJIRMS) Volume 03 Issue 12 Dec 2018, ISSN: 2455-8737, Imp. Factor - 4.102 Available online at - $\underline{w w w . j j i r m s . i n}$}

comparable to this study.The overall clinical diagnostic in Chamyal and Sabargirish study was $88.3 \%$ which is comparable to the present study. ${ }^{[22]}$

In the study of Ramani et.al., ${ }^{[23]}$ shows that Hodgkin"s lymphoma $25 \%$ found the median age of presentation of Hodgkin"s lymphoma in India as 34 years which is lower than that seen in the west which agree with the findings of siddiqui et.al., ${ }^{[24]}$ where male were predominance which was quite more than this study.

For diagnosis histopathological examination and open biopsy is the gold standard Jha B.C., et al. ${ }^{[4]}$ reported a sensitivity of $92.8 \%$ tuberculous lymphadenitis. Dandapat M.C., et al. ${ }^{[10]}$ shows a sensitivity of $83 \%$ for tuberculosis. Mondal A., et al. ${ }^{[12]}$ reported $100 \%$ sensitivity in tuberculous and phylogenic lymphadenitis, and Hodgkin's disease, 98\% for metastatic deposits; 97\% for chronic non-specific lymphadenitis; $92 \%$ for Non Hodgkin's lymphomas, ${ }^{[25,26]}$ which is quite similar to this study.

From this study, it was noticed that FNAC is an one of the most important diagnostic tool for diagnosis Lymphadenopathy with very cost effective. It is easily and safely done. From all the above observations a protocol diagnosis of Lymphadenopathy cases can be formulated for evaluation.

\section{Conclusion}

Lymphadenopathy is a clinical manifestation of systemic as well as regional disease. It can be in the form of malignant and benign causes. In this study, it was found that tuberculosis is the common cause of Lymphadenopathy, followed by chronic non-specific lymphadenitis. In lymph nodes benign lesions are common than malignant lesions. Younger age group are more affected by tuberculosis whereas, malignancies are affect in older age groups.Posterior triangle nodes are common group of lymph nodes affected in tuberculosis followed by jugulodi gastric nodes. It can be concluded that Lymphadenopathy is a clinical evaluation followed by FNAC which is reliable diagnostic tool that is easy to perform, cost effective, speedy results accurate can be obtained. Specialist input is the main requirement for this. Biopsy is also useful in cases of lymphomas that act as diagnostic tool. When FNAC report is inconclusive, in nonspecific lymphadenitis than Biopsy is much helpful for accurate diagnosis and management. Hence this study is very helpful for the patients from backward area and help in appropriate management with minimal investigative procedures.It also creates an awareness regarding tubercular lymphadenitis as well as other lymphadenitis in this area.

For lymphadenitis, lymphomas and secondary deposits FNAC (fine needle aspiration cytology) has higher accuracy. For diagnosis of chronic non-specific lymphadenitis it has lesser accuracy. Hence, in cases of Lymphadenopathy FNAC was found to be reliable, simple and cost effective method for diagnosis.

\section{References}

[1] Shafiullah, Syed H, et al. Tuberculous lymphadenitis on Afghan refugees. J Pathol., 1999; 187: 28-38.

[2] Robbins and Cotran Pathological basis of Disease 8th edtn
[3] Abramowitz I. "Lymph tissue of the Head and Neck", in Lee McGregor"s synopsis of surgical anatomy, 12th Edition. John Wright and Sons,1999;193-195.

[4] Jha B C, Dass A. Cervical Tuberculous lymphadenopathy: changing clinical patterns and concepts in management. Post- graduate Med. J, 2001; 77(905): 185-7.

[5] Jindal n, Devi B, Aggarwal A. Mycobacterial cervical lymphadenopathy in childhood. Post-graduate Med. J, 2002; 87: 182-3.

[6] Nataraj G, Kurup S, Pandit A, Mehta P. Correlation of FNAC, smear and culture intubercular lymphadenitis: a prospective study. Indian J Pathol., 2001; 82: 96-97.

[7] Arora B, Arora DR. FNAC in diagnosis of tubercular lymphadenitis. Indian J Medical Research, 1990; 91: 189-92.

[8] Kim LH, Peh SC, Chen KS. Pattern of lymphnode Pathology in a private laboratory. Malays J Pathol., 1999; 21(2): 87-93.

[9] Aruna D, Mahopatro S. Correlation of FNAC with histopathological study in peripheral lymph node lesions. Indian J PatholMicrobiol., 1999; 30(2): 96-98.

[10] Dandapat MC, Mishra BM, Dash SP, Kar PK. Peripheral lymph node tuberculosis: a review of 80 cases. $\mathrm{Br} \mathrm{J}$ Surg., 1990 Aug; 77(8): 911-12

[11] Dasgupta A, Ghosh RN, Poddar AK. FNAC of cervical lymph nodes with special reference to tuberculosis. J Indian Med Assoc, 1994 Feb; 92(2): 44-6.

[12] Mondal A, Mukherjee D, Chatterjee DN. FNAC in diagnosis of cervical lymphadenopathy. J Ind Med Assoc., 1989 Dec; 87(2): 281-3

[13] Prasad RR, Narasimhan R. Shankaran V. FNAC in diagnosis of superficial Lymphadenopathy: an analysis of 2418 cases. Diagcytopathol., 1996 Dec; 15(5): 382-6.

[14] Gupta AK, Nayar M, Chandra M.Critical appraisal of fine needle aspiration cytology in tuberculouslymphadenitis.Acta Cytol.1992;36(3):391-4.

[15] Pandit A, Candes FP, Khubchandani SR. Fine needle aspiration cytology of Lymph node., Journal of Postgraduate Medicine 1987;33:134-136

[16] Rakhshan M, RakhshanA.The diagnostic accuracy of fine needle aspiration cytology in Neck lymphoid masses. Iranian J Pathol 2008; 4:147-50.

[17] Narang RK, Pradhan S, Singh RP, Chaturvedi S. Place of fine needle Aspiration cytology in the diagnosis of Lymphadenopath. Indian Journal of Surgery 1990;37:2931 .

[18] Khan RA, Wahab S, Chana RS, Naseem S, Siddique S. Children with significant cervical lymphadenopathy: clinicopathological analysis and role of fine-needle aspiration in Indian setup. J Pediatr (Rio J). 2008;84(5):449-454

[19] Desai HV, Daxini AB, Pandey AS, Raval VK, ModhDA.Clinical profile of patients with tubercular lymphadenitis.Int J Res Med. 2015; 4(4);22-27 DandapatDandapat, M.C. Panda, B.K., Patra, A.K. and Acharya, N. Diagnosis of tubercular lymphadenitis by Fine Needle Aspiration Cytology. Ind. J. Tub.1987, 34,139-142.

[20] Ahmed N, Israr S, Ashraf MS. Comparison of fine needle aspiration cytology and excision biopsy in the 
diagnosis of cervical lymphadenopathy. Pak J Surg. 2009;25(2):72-5

[21] Hirachand S, Lakhey M, Akhter J, Thapa B. Evaluation of fine needle aspiration cytology of lymph nodes in Kathmandu Medical College, Teaching hospital. Kathmandu Univ Med J. 2009;7(26):139-42.

[22] Chamyal PC, Sabarigirish K. Clinico pathological correlation study of cervical lymph node masses. Int $\mathbf{J}$ Otolaryngol Head Neck Surg. 1997;49(4);404-5.

[23] Ramani A, Kumar KA, Rao KK, Vidyasagar MS, Kandaje GN. A clinicopathological Profile of lymphomas in South India. Journal Association Physician of India 1992;40(4):282-283
[24] Siddiqui N, Ayub B, Badar F, ZaidiA.Hodgkin's lymphoma in Pakistan: a clinico-epidemiological study of 658 cases at a cancer center in Lahore.Asian Pac J Cancer Prev. 2006;7(4): 651-655

[25] Hewitt HB, Blake ER. Further studies of the relationship between lymphatic dissemination and lymph nodal metastasis in a non immugenic murine tumors. Br. J Cancer, 2006; 35: 45.

[26] Ammari FF, Bani Hani AH. Tuberculosis of lymph glands of neck:a limited role for surgery. Otolaryngol Head and Neak Surgery, 2003; 128(4): 576-80. 